Article

\title{
A Multicountry Ecological Study of Cancer Incidence Rates in 2008 with Respect to Various Risk-Modifying Factors
}

\author{
William B. Grant \\ Sunlight, Nutrition, and Health Research Center, P.O. Box 641603, San Francisco, CA 94164-1603, \\ USA; E-Mail: wbgrant@infionline.net; Tel.: +1-415-409-1980
}

Received: 6 November 2013; in revised form: 12 December 2013 / Accepted: 14 December 2013 / Published: 27 December 2013

\begin{abstract}
Observational and ecological studies are generally used to determine the presence of effect of cancer risk-modifying factors. Researchers generally agree that environmental factors such as smoking, alcohol consumption, poor diet, lack of physical activity, and low serum 25-hdyroxyvitamin D levels are important cancer risk factors. This ecological study used age-adjusted incidence rates for 21 cancers for 157 countries (87 with high-quality data) in 2008 with respect to dietary supply and other factors, including per capita gross domestic product, life expectancy, lung cancer incidence rate (an index for smoking), and latitude (an index for solar ultraviolet-B doses). The factors found to correlate strongly with multiple types of cancer were lung cancer (direct correlation with 12 types of cancer), energy derived from animal products (direct correlation with 12 types of cancer, inverse with two), latitude (direct correlation with six types, inverse correlation with three), and per capita gross national product (five types). Life expectancy and sweeteners directly correlated with three cancers, animal fat with two, and alcohol with one. Consumption of animal products correlated with cancer incidence with a lag time of 15-25 years. Types of cancer which correlated strongly with animal product consumption, tended to correlate weakly with latitude; this occurred for 11 cancers for the entire set of countries. Regression results were somewhat different for the 87 high-quality country data set and the 157-country set. Single-country ecological studies have inversely correlated nearly all of these cancers with solar ultraviolet-B doses. These results can provide guidance for prevention of cancer.
\end{abstract}

Keywords: cancer incidence rates; diet; ecological; latitude; smoking; ultraviolet-B; vitamin D 


\section{Introduction}

Cancer is one of the leading causes of disease and death worldwide. In 2008, an estimated 12.66 million new cases of cancer occurred, excluding nonmelanoma skin cancer, and 7.56 million cancer deaths (approximately 13\% of all deaths) [1]. The most frequent types were lung, prostate, colorectal, stomach, and liver cancer for men, and breast, colorectal, cervix uteri, lung, and stomach cancer for women. However, in the United States in 2011, cancer deaths accounted for $23 \%$ of all deaths $[2,3]$.

Although much of the war on cancer emphasizes early detection and treatment, the burden of cancer will remain high unless the primary risk factors for cancer are understood and addressed. A general understanding exists of many of the cancer risk-modifying factors; for example, according to the World Health Organization (WHO) [4].

About $30 \%$ of cancer deaths are due to the five leading behavioral and dietary risks - high body mass index, low fruit and vegetable intake, lack of physical activity, tobacco use, and alcohol use.

- Tobacco use is the most important risk factor for cancer, causing $22 \%$ of global cancer deaths and $71 \%$ of global lung cancer deaths.

- Cancer-causing viral infections such as hepatitis B and $\mathrm{C}$ viruses and human papillomavirus are responsible for up to $20 \%$ of cancer deaths in low- and middle-income countries.

The American Institute for Cancer Research/World Cancer Research Fund [5] recommends limiting intake of red meat and avoiding processed meat, eating mostly foods of plant origin, and limiting consumption of energy-dense foods, which includes sugary drinks.

Several epidemiological approaches are used to investigate cancer risk-modifying factors. These approaches include case-control studies, nested case-control studies derived from cohort studies, and ecological studies. Multicountry ecological studies have been used to identify and quantify risk factors for cancer. In 1975, examined dietary links to cancer by using incidence data for 27 cancers from 23 countries and mortality rates for 14 cancers from 32 countries [6]. They found that meat consumption was an important risk factor for colon cancer and that fat consumption was an important risk factor for breast and endometrial cancer. Per capita gross domestic product (GDP) also highly correlated with these cancers.

Both single- and multicountry ecological studies offer evidence that solar ultraviolet-B (UVB) irradiance, through production of vitamin D, reduces cancer risk [7-10]. The UVB-vitamin D-cancer hypothesis has received reasonable support in other types of studies, including case-control and nested case-control studies, and the mechanisms whereby vitamin $\mathrm{D}$ reduces risk of cancer are well known [11,12]. The UVB-vitamin D-cancer hypothesis is thought to generally satisfy the criteria for causality in a biological system outlined by Hill [13] for several cancers [14,15].

Using the ecological study approach, this study presents results with cancer incidence data for 87 countries with high-quality mortality rate data and 157 countries total with respect to dietary supply and other factors, including per capita GDP, life expectancy, smoking, and latitude (an index for solar UVB doses). I then compare the results to published results from observational studies. 


\section{Data and Methods}

The hypothesis tested in this study is that various cancer risk-modifying factors affect cancer incidence rates at the national population level and the relative contributions to cancer risk can be determined. The risk-modifying factors considered in this study include alcohol consumption, animal fat consumption, animal product consumption, cereals consumption, gross domestic product, life expectancy, solar UVB and vitamin D production, and sweeteners consumption. Other components of diet, such as eggs, fish, meat, and milk, were considered, but since they are highly correlated with total animal product supply, it was not though that any one of these factors would alone explain the finding for total animal product supply. Other cancer risk-modifying factors were not considered; generally due to the fact that it would be difficult to obtain or develop and index that could be used for all countries. Some of the factors omitted are discussed later.

Cancer incidence data for 2008 came from GLOBOCAN [1]. I chose countries used in this study according to two criteria. First, the country must have dietary supply data from the Food and Agriculture Organization published in printed form for 1992-1994 [16]. The reason for this requirement is that diet may affect the risk of cancer 10-30 years before cancer develops [17,18]. Second, the country must have more than 100,000 inhabitants in 1992-1994 since developing robust cancer statistics for countries with fewer inhabitants is difficult. A total of 157 countries met both criteria. Of those, 87 countries have level 1 or 2 quality data (hereafter, high-quality data), on the basis of classifications by WHO for deaths in 2001-2002 [19,20]. Appendix lists countries used in this study.

Dietary supply data came from the Food and Agriculture Organization, both from the book published in 1996 for 3-year averaged data from 1979-1981 to 1992-1994 [16] and from their website for single-year averaged data for 1995 and 2000 [21]. I obtained values for alcohol, animal fat, animal product energy, cereals, energy (total), fat, fish, and meat. I used data for various years since a 10- to 30 -year lag generally exists between national dietary changes and cancer incidence rate $[17,18]$. For example, animal product energy supply increased in the 1990s for some countries but decreased in other countries; varying the year of the data serves to help estimate the lag between dietary change and cancer incidence.

I used lung cancer incidence rates as the index of health effects of smoking. Ecological studies of cancer in Nordic countries have used lung cancer incidence rates [22], and ecological studies in the United States [8] and Spain [23] have used lung cancer mortality rates. Lung cancer incidence or mortality rates are ideal since they integrate the effects of smoking over many years [24]. Also, cigarette consumption data are not available for all countries. A possible downside is that diet and other factors, such as solar UVB doses/vitamin D [25], can also affect risk of lung cancer. However, as results for various cancers will show, this does not present a problem.

Nonetheless, I obtained data for cigarette consumption per capita for 1990 from WHO, generally of the form used on its data sheet for Poland [26], with the country name changed for different countries. Some URLs were of a slightly different form and found through additional Internet searches. I chose the year 1990 since lung cancer takes many years to develop and I judged the data for 2000 to be too recent. I could have used data for 1980 although fewer countries have data for 1980, but the data for 1990 serve the intended purpose to show that lung cancer is strongly linked to cigarette consumption. I obtained data for 71 countries, including 54 countries with high-quality cancer incidence data and life 
expectancy greater than 60 years for males in 1990. I apportioned the cigarette consumption data to males and females in proportion to the male-female balance of lung cancer incidence rates in each country in 2008 .

The index used for socioeconomic status is GDP per capita for 1999 from the International Monetary Fund [27]. Data were available for 143 countries. However, the analyses omitted data for one country, Brunei, since the high per capita GDPs for this country were acquired recently and probably have not been shared with many in the country's population, such as guest workers. I considered two other sets of data: International Monetary Fund data for 1989 [27] and CIA values for 2008 [28]. Among concerns regarding the 1989 data is that many countries were not included, in part since they were not independent countries then, and that 1989 was 19 years before the cancer incidence data. One problem with the 2008 data is that the data are for the same year as for cancer incidence rates. However, GDP per capita for the year of cancer incidence could reflect cancer screening rates for breast, colorectal, and prostate cancer.

The analyses also used latitude of the approximate center of population of each country. This index is considered an index of annual solar UVB doses.

I obtained life expectancy data for males in 1990 for 151 countries from World Health Statistics [19]. For some cancers, cancer rates correlated with life expectancy for countries with life expectancy greater than 60 years.

I analyzed the countries in three different sets: the entire set (157 countries); that for countries with life expectancy of 60 years or greater for males in 1990 (100 countries); and countries with high-quality data in 2001 and 2002 for causes of death according to [15,19], along with life expectancy greater than 60 years for males in 1990 ( 87 countries). I estimated cancer incidence rates for the remaining 70 countries from data for countries with high-quality data [20]. Thus, rates are not entirely independent of rates in countries with high-quality data; however, values for risk-modifying values should be independent. When the incidence rate was zero, I omitted the value. KaleidaGraph [29] created the graphs.

I ran regression analyses by using IBM SPSS Statistics 20 (Armonk, New York, NY, USA). The analyses included various combinations of risk-modifying factors for each type of cancer and for both sexes. I eliminated those factors with large $p$ values and reran the regression. For factors that had two or more sets of data for various years, such as animal products, cereals, and sweeteners, I used pairs of data to see which had the stronger correlation. This was a useful step since not all countries had values for each year. When multiple linear regression analysis found significantly correlated factors, I ran each factor in a linear regression analysis to see whether each was independently significantly correlated with cancer incidence rates and with the same direction (direct or inverse) since the factors are highly correlated and can interfere with each other. The final analysis omitted those not independently correlated.

Table 1 lists cross-correlation coefficients for various risk-modifying factors for the 87 countries with high-quality data. Most factors are highly correlated with each other, except sweeteners. Latitude is highly correlated with energy from animal products $(0.74<r<0.79)$. Cereals are inversely correlated with all other factors. 


\section{Results}

Table 2 gives regression results for the set of 87 high-quality countries. Significant results emerged for 21 cancers as well as all and all less lung cancer for countries with high-quality data. The regression found no significant results for esophageal, gallbladder, and stomach cancer.

Table 3 gives regression results for all 157 countries. The regression results for the 100 countries with male life expectancy greater than 60 years in 1990 were similar to those for the other two sets of countries (data not shown).

Table 4 gives the numbers of cancers significantly correlated with each risk-modifying factor for the two sets of countries. Four factors have significant correlations with a reasonable number of cancer types: lung cancer, energy derived from animal products, latitude, and GDP. Alcohol supply is associated only with colorectal cancer.

The results for animal product energy show that data for 1990 have the most high-significance correlations with cancer incidence, indicating about a 15- to 20-year lag between diet and cancer incidence. Prostate cancer has the longest lag, 28 years.

Life expectancy is significantly associated with several cancers: brain and colorectal cancer, leukemia, and multiple myeloma.

Latitude is directly correlated with several cancers: bladder, brain, kidney, lung (females), melanoma, and Hodgkin's lymphoma, and inversely correlated with cervical, lip, and thyroid cancer. Latitude is generally not found significantly correlated with cancer incidence rates if animal product energy is; this is the case for 11 cancers. Both animal product energy and latitude were significantly correlated for only two cancers: brain and kidney cancer. Evidently when energy from animal products is significantly correlated with cancer incidence rates, it overwhelms the effect of latitude.

Lung cancer was directly correlated with cigarette consumption per capita for both sexes as well as animal fat in 1993 and latitude.

The regression results for all cancer less lung cancer for both males and females for high-quality countries found a significant correlation between energy derived from animal products and lung cancer. Alcohol and latitude were also directly correlated with all cancer less lung cancer for females, whereas cereals were inversely correlated.

\section{Discussion}

The lag of 15-20 years between energy derived from animal products is supported from studies of cancer trends in countries undergoing nutrition transitions: 15-27 years for colon cancer mortality rates in Japan [30], 20-31 years for breast cancer mortality rates in Japan [18], and 10 years for mortality rates for several cancers in several Southeast Asian countries [19]. Studies reporting early-life effects on risk of prostate cancer [31,32] support the finding of 28 years for prostate cancer.

Other evidence supporting the role of energy derived from animal products as important risk factors includes the finding that Seventh Day Adventists, who generally are lacto-ovo vegetarians who do not smoke or drink alcohol excessively, have lower cancer rates than do others living in the same region [33-35]. 
Table 1. Cross-correlations of factors used in this study for the 87 high-quality countries *.

\begin{tabular}{|c|c|c|c|c|c|c|c|}
\hline Factor & $\begin{array}{c}\text { Animal Energy, } \\
1980 \\
\end{array}$ & $\begin{array}{c}\text { Animal Energy, } \\
1985\left(r, r^{2}, p\right)\end{array}$ & $\begin{array}{c}\text { Animal Energy, } \\
1990\left(r, r^{2}, p\right)\end{array}$ & $\begin{array}{c}\text { Animal Energy, } \\
1993\left(r, r^{2}, p\right)\end{array}$ & $\begin{array}{c}\text { Animal Energy, } \\
2000\left(r, r^{2}, p\right)\end{array}$ & $\begin{array}{l}\text { Animal Fat, } \\
2000\left(r, r^{2}, p\right)\end{array}$ & $\begin{array}{c}\text { Cereals } \\
1993\left(r, r^{2}, p\right) \\
\end{array}$ \\
\hline Alcohol, 1993 & $0.62,0.37$ & $0.66,0.43$ & $0.71,0.49$ & $0.63,0.38$ & $0.65,0.41$ & $0.67,0.44$ & $-0.51,0.24$ \\
\hline Animal en, 1980 & & $0.99,0.98$ & $0.96,0.92$ & $0.94,0.87$ & $0.94,0.87$ & $0.92,0.85$ & $-0.59,0.34$ \\
\hline Animal en, 1985 & & & $0.98,0.96$ & $0.96,0.92$ & $0.94,0.88$ & $0.95,0.90$ & $-0.61,0.36$ \\
\hline Animal en, 1990 & & & & $0.99,0.97$ & $0.95,0.89$ & $0.95,0.90$ & $-0.61,0.36$ \\
\hline Animal en, 1993 & & & & & $0.95,0.91$ & $0.95,0.90$ & $-0.58,0.33$ \\
\hline Animal en 2000 & & & & & & $0.99,0.98$ & $-0.60,0.36$ \\
\hline Animal fat, 2000 & & & & & & & $-0.60,0.35$ \\
\hline Factor & $\begin{array}{c}\text { Fat, } 2000 \\
\left(r, r^{2}, p\right)\end{array}$ & $\begin{array}{c}\text { GDP } 1999 \\
\left(r, r^{2}, p\right)\end{array}$ & $\begin{array}{c}\text { Latitude } \\
\left(r, r^{2}, p\right)\end{array}$ & $\begin{array}{c}\text { Life Expectancy } \\
\quad\left(r, r^{2}, p\right)\end{array}$ & $\begin{array}{c}\text { Lung Cancer, } M \\
\quad\left(r, r^{2}, p\right)\end{array}$ & $\begin{array}{c}\text { Lung Cancer } \\
\mathrm{F}\left(r, r^{2}, p\right)\end{array}$ & $\begin{array}{l}\text { Sweeteners } 1990 \\
\quad\left(r, r^{2}, p\right)\end{array}$ \\
\hline Alcohol, 1993 & $0.63,0.39$ & $0.57,0.31$ & $0.51,0.25$ & $0.47,0.21$ & $0.53,0.27$ & $0.45,0.19$ & $0.13,0.001,0.30$ \\
\hline Animal en, 1980 & $0.81,0.64$ & $0.74,0.54$ & $0.79,0.63$ & $0.63,0.38$ & $0.61,0.36$ & $0.73,0.53$ & $0.29,0.07,0.02$ \\
\hline Animal en, 1985 & $0.84,0.69$ & $0.77,0.59$ & $0.79,0.61$ & $0.66,0.43$ & $0.63,0.39$ & $0.72,0.51$ & $0.28,0.07,0.02$ \\
\hline Animal en, 1990 & $0.85,0.72$ & $0.76,0.57$ & $0.77,0.60$ & $0.67,0.43$ & $0.65,0.42$ & $070,0.49$ & $0.23,0.04,0.06$ \\
\hline Animal en, 1993 & $0.85,0.72$ & $0.75,0.55$ & $0.67,0.44$ & $0.65,0.42$ & $0.51,0.25$ & $0.65,0.41$ & $0.17,0.02,0.16$ \\
\hline Animal en 2000 & $0.89,0.79$ & $0.79,0.62$ & $0.66,0.43$ & $0.66,0.43$ & $0.51,0.25$ & $0.67,0.45$ & $0.17,0.02,0.16$ \\
\hline Animal fat, 2000 & $0.89,0.78$ & $0.77,0.59$ & $0.66,0.43$ & $0.63,0.39$ & $0.51,0.25$ & $0.67,0.45$ & $0.17,0.01,0.18$ \\
\hline Cereals, 1993 & $-0.53,0.27$ & $-0.58,0.33$ & $-0.14,0.008,0.19$ & $-0.62,0.38$ & $-0.23,0.04,0.04$ & $-0.50,0.24$ & $-0.35,0.11,0.003$ \\
\hline Fat, 2000 & & $0.83,0.68$ & $0.53,0.27$ & $0.73,0.53$ & $0.46,0.20$ & $0.65,0.41$ & $0.14,0.004,0.27$ \\
\hline GDP, 1999 & & & $0.41,0.16$ & $0.77,0.59$ & $0.26,0.06,0.02$ & $0.67,0.44$ & $0.37,0.12,0.003$ \\
\hline Latitude & & & & $0.24,0.05,0.02$ & $0.63,0.39$ & $0.47,0.21$ & - \\
\hline Life expectancy & & & & & $0.30,0.08,0.005$ & $0.55,0.29$ & $0.29,0.07,0.02$ \\
\hline Lung cancer, M, 2008 & & & & & & $0.49,0.23$ & - \\
\hline Lung cancer, F, 2008 & & & & & & & $0.32,0.09,0.007$ \\
\hline
\end{tabular}

—: no significant results; *: if $p<0.001$, value omitted; F: female; M: male; Animal en, Animal energy. After obtaining results of the regression analyses, I searched the journal literature through PubMed.gov for papers that supported the findings for risk-modifying factors for each type of cancer. The search was not meant to be comprehensive or to provide enough information to conclude whether the support could be considered definitive. 
Table 2. Regression results for 87 high quality countries.

\begin{tabular}{|c|c|c|c|c|c|c|c|c|}
\hline Cancer & Sex & $\begin{array}{c}\text { Number } \\
\text { of } \\
\text { Countries }\end{array}$ & $\begin{array}{c}\text { Lung Cancer } \\
\qquad(\beta, p)\end{array}$ & $\begin{array}{c}\text { Animal Energy } \\
\quad(\text { year; } \beta, p)\end{array}$ & $\begin{array}{l}\text { Latitude } \\
\qquad(\beta, p)\end{array}$ & $\begin{array}{l}\text { GDP } 1999 \\
\quad(\beta, p)\end{array}$ & $\begin{array}{l}\text { Miscellaneous } \\
\qquad(\beta, p)\end{array}$ & $\begin{array}{c}\text { Adjusted } \\
r^{2}, F, p\end{array}$ \\
\hline \multirow[t]{4}{*}{ All } & $\mathrm{M}$ & 82 & $0.40, *$ & $2000 ; 0.44, *$ & & & Alc93: $0.23,0.003$ & $0.77,93, *$ \\
\hline & & 84 & $0.38, *$ & $1993 ; 0.46, *$ & & & Alc93: 0.20, 0.997 & $0.77,91, *$ \\
\hline & $\mathrm{F}$ & 87 & $0.49, *$ & $1993 ; 0.33, *$ & & & Cer93: $-0.20,0.002$ & $0.77,99, *$ \\
\hline & & 82 & $0.53, *$ & $1993 ; 0.30, *$ & & & Alc93: 0.30, 0.004 & $0.77,93, *$ \\
\hline \multirow[t]{5}{*}{ All Less Lung } & M & 62 & $0.23,0.01$ & $1980 ; 0.54, *$ & & $0.21,0.04$ & & $0.74,58, *$ \\
\hline & & 87 & $0.28, *$ & $1993 ; 0.52, *$ & & & Cer93: $-0.21,0.006$ & $0.69,63, *$ \\
\hline & $\mathrm{F}$ & 62 & $0.40, *$ & $1990 ; 0.44, *$ & & $0.10,0.38$ & & $0.72,52, *$ \\
\hline & & 82 & $0.43, *$ & $1993 ; 0.34, *$ & & & Alc93: 0.22, 0.004 & $0.71,67, *$ \\
\hline & & 82 & $0.43, *$ & & $0.22,0.004$ & & $\begin{array}{l}\text { Alc93: } 0.20,0.013 \\
\text { Cer00: }-0.25,0.001\end{array}$ & $0.71,51, *$ \\
\hline \multirow[t]{2}{*}{ Bladder } & M & 79 & $0.43, *$ & & $0.30,0.003$ & $0.23,0.006$ & & $0.58,37, *$ \\
\hline & $\mathrm{F}$ & 80 & $0.48, *$ & & $0.26,0.003$ & $0.17,0.09$ & & $0.58,37, *$ \\
\hline \multirow[t]{3}{*}{ Brain } & M & 79 & & & $0.58, *$ & $0.26,0.004$ & & $0.52,44, *$ \\
\hline & & 79 & & $1993 ; 0.26,0.004$ & $0.58, *$ & & & $0.51,41, *$ \\
\hline & $\mathrm{F}$ & 85 & $0.33,0.001$ & & $0.38, *$ & & & $0.35,24, *$ \\
\hline Breast & $\mathrm{F}$ & 80 & $0.23,0.009$ & $1993 ; 0.41, *$ & & $0.31,0.002$ & & $0.72,67, *$ \\
\hline Cervix & $\mathrm{F}$ & 80 & & & $-0.40, *$ & $-0.33,0.001$ & & $0.37,24, *$ \\
\hline \multirow[t]{3}{*}{ Colorectal } & M & 77 & $0.40, *$ & & & $0.31, *$ & Alc93: 0.39, * & $0.75,76, *$ \\
\hline & $\mathrm{F}$ & 67 & $0.37, *$ & $1985 ; 0.17,0.12$ & & & $\begin{array}{l}\text { Alc93: } 0.24,0.003 \\
\text { LE: } 0.28,0.001\end{array}$ & $0.77,56, *$ \\
\hline & & 82 & $0.33, *$ & & $0.25,0.001$ & & $\begin{array}{c}\text { Alc93: } 0.25,0.001 \\
\text { LE: } 0.28, *\end{array}$ & $0.74,58, *$ \\
\hline Corpus uteri & $\mathrm{F}$ & 68 & & $1985 ; 0.67, *$ & & & & $0.44,53, *$ \\
\hline
\end{tabular}


Table 2. Cont.

\begin{tabular}{|c|c|c|c|c|c|c|c|c|}
\hline \multirow[t]{2}{*}{$\begin{array}{l}\text { Hodgkin's } \\
\text { Lymphoma }\end{array}$} & M & 82 & & & $0.25,0.02$ & & Fat00: $0.48, *$ & $0.40,28, *$ \\
\hline & $\mathrm{F}$ & 78 & & & $0.33,0.005$ & & Fat00: $0.29,0.01$ & $0.26,15, *$ \\
\hline \multirow[t]{5}{*}{ Kidney } & M & 87 & $0.27,0.004$ & & $0.50, *$ & & Cer93: $-0.25,0.001$ & $0.60,44, *$ \\
\hline & & 87 & $0.27,0.003$ & $1993 ; 0.32,0.001$ & $0.32,0.003$ & & & $0.60,43, *$ \\
\hline & & 87 & $0.40, *$ & $1993 ; 0.47, *$ & & & & $0.56,55, *$ \\
\hline & $\mathrm{F}$ & 85 & $0.30,0.001$ & & $0.52, *$ & & Cer93: $-0.17,0.04$ & $0.60,42, *$ \\
\hline & & 85 & $0.30,0.002$ & $1993 ; 0.23,0.04$ & $0.39, *$ & & & $0.60,42, *$ \\
\hline \multirow[t]{2}{*}{ Laryngeal } & M & 85 & $0.86, *$ & $2000 ;-0.33, *$ & & & & $0.55,52, *$ \\
\hline & $\mathrm{F}$ & 78 & $0.48,0.001$ & $2000 ;-0.39,0.008$ & & & & $0.12,6, *$ \\
\hline \multirow[t]{3}{*}{ Leukemia } & M & 86 & $0.43, *$ & & & & LE: $0.44, *$ & $0.47,39, *$ \\
\hline & & 80 & $0.46, *$ & & & $0.40, *$ & & $0.46,35, *$ \\
\hline & $\mathrm{F}$ & 86 & $0.30,0.006$ & & & & LE: $0.36,0.001$ & $0.32,21 *$ \\
\hline \multirow[t]{2}{*}{ Lip, oral } & M & 87 & $0.53, *$ & & & & & $0.28,34, *$ \\
\hline & $\mathrm{F}$ & 86 & $0.51, *$ & & $-0.31,0.007$ & & & $0.19,11, *$ \\
\hline \multirow[t]{2}{*}{ Liver } & M & 67 & & $1980 ;-0.27,0.03$ & & & & $0.06,5,0.03$ \\
\hline & $\mathrm{F}$ & 67 & & $1980 ;-0.51, *$ & & & & $0.25,23, *$ \\
\hline \multirow[t]{9}{*}{ Lung } & M & 54 & CigM: $0.65, *$ & & & & AF93; 0.36, * & $0.73,72, *$ \\
\hline & & 54 & CigM: $0.63, *$ & & $0.35, *$ & & & $0.72,67, *$ \\
\hline & & 54 & CigM: $0.79, *$ & & & & & $0.62,89, *$ \\
\hline & & 54 & Cig: $0.80, *$ & & & & & $0.62,86, *$ \\
\hline & $\mathrm{F}$ & 54 & CigF: $0.65, *$ & & & & AF93; 0.32,* & $0.77,92, *$ \\
\hline & & 54 & CigF: $0.64, *$ & & $010,0.35$ & & AF93: $0.26,0.018$ & $0.77,61, *$ \\
\hline & & 54 & CigF: $0.70, *$ & & $0.26,0.003$ & & & $0.75,81, *$ \\
\hline & & 54 & CigF: $0.85, *$ & & & & & $0.71,131, *$ \\
\hline & & & & & Lat $\times$ Lat & & & \\
\hline
\end{tabular}


Table 2. Cont.

\begin{tabular}{|c|c|c|c|c|c|c|c|c|}
\hline Melanoma & $\mathrm{M}$ & $53 * *$ & & & $0.41, *$ & $0.52, *$ & & $0.72,68, *$ \\
\hline & & $54 * * *$ & & & $0.48, *$ & $0.43, *$ & & $0.69,59, *$ \\
\hline & & $52 * *$ & & & $0.40, *$ & $0.53, *$ & & $0.72,67, *$ \\
\hline & & $53 * * *$ & & & $0.45, *$ & $0.47, *$ & & $0.70,63, *$ \\
\hline \multirow[t]{4}{*}{$\begin{array}{l}\text { Multiple } \\
\text { myeloma }\end{array}$} & $\mathrm{M}$ & 64 & & $85 ; 0.57, *$ & & & LE: $0.27,0.02$ & $0.59,46, *$ \\
\hline & & 58 & & $85 ; 0.53, *$ & & $0.29,0.04$ & & $0.59,42, *$ \\
\hline & $\mathrm{F}$ & 77 & & & & $0.44, *$ & LE: $0.37,0.003$ & $0.58,54, *$ \\
\hline & & 77 & & & & $0.74, *$ & & $0.53,88, *$ \\
\hline \multirow[t]{2}{*}{$\begin{array}{c}\text { Non-Hodgkin's } \\
\text { lymphoma }\end{array}$} & $\mathrm{M}$ & 80 & & & & $0.75, *$ & & $0.55,97, *$ \\
\hline & $\mathrm{F}$ & 80 & & & & $0.73, *$ & & $0.54,91, *$ \\
\hline Ovarian & $\mathrm{F}$ & 68 & & $85 ; 0.70, *$ & & & & $0.49,65, *$ \\
\hline \multirow[t]{3}{*}{ Pancreatic } & $\mathrm{M}$ & 86 & $0.71, *$ & $1993 ; 0.16,0.04$ & & & & $0.64,78, *$ \\
\hline & & 86 & $0.80, *$ & & & & & $0.63,144, *$ \\
\hline & $\mathrm{F}$ & 86 & $0.40,0.001$ & $1993 ; 0.31,0.008$ & & & & $0.39,29, *$ \\
\hline Prostate & $\mathrm{M}$ & 68 & $-0.26,0.014$ & $80 ; 0.62, *$ & & & $\begin{array}{c}\text { Cer93: }-0.36, \\
0.001\end{array}$ & $0.59,33, *$ \\
\hline Testicular & $\mathrm{M}$ & 59 & & $85 ; 0.66, *$ & & $0.25,0.023$ & & $0.74,82, *$ \\
\hline \multirow[t]{2}{*}{ Thyroid } & $\mathrm{M}$ & 82 & & $1993 ; 0.74, *$ & $-0.29,0.023$ & & & $0.33,20, *$ \\
\hline & $\mathrm{F}$ & 84 & & $1993 ; 0.58, *$ & $-0.25,0.07$ & & & $0.19,10, *$ \\
\hline
\end{tabular}

*: $p<0.001$; **: omits Australia, Luxembourg, New Zealand; **: omits Australia, New Zealand; AF93: animal fat, 1993; Alc93: alcohol supply, 1993; Cer: cereals supply; Cer90: cereals supply, 1990; LE: life expectancy; F: female; M: male; CigF: cigarettes consumed by females; CigM: cigarettes consumed by males; Fat00: Fat, 2000. 
Table 3. Regression results for the 157 countries.

\begin{tabular}{|c|c|c|c|c|c|c|c|c|c|}
\hline Cancer & Sex & $\begin{array}{c}\text { Number } \\
\text { of } \\
\text { Countries }\end{array}$ & $\begin{array}{c}\text { Lung Cancer } \\
\qquad(\beta, p)\end{array}$ & $\begin{array}{l}\text { Animal Energy } \\
(\text { year; } \beta, p)\end{array}$ & $\begin{array}{l}\text { Latitude } \\
\qquad(\beta, p)\end{array}$ & $\begin{array}{c}\text { GDP } 1999 \\
(\beta, p)\end{array}$ & $\begin{array}{c}\text { Miscellaneous } \\
(\beta, p)\end{array}$ & $\begin{array}{c}\text { Miscellaneous } \\
(\boldsymbol{\beta}, \boldsymbol{p})\end{array}$ & $\begin{array}{c}\text { Adjusted } \\
r^{2}, F, p\end{array}$ \\
\hline \multirow[t]{2}{*}{ Bladder } & $\mathrm{M}$ & 140 & $0.40, *$ & & $0.36, *$ & $0.19,0.002$ & & & $0.66,91, *$ \\
\hline & $\mathrm{F}$ & 139 & $0.37, *$ & & $0.25,0.001$ & $0.22,0.009$ & & & $0.52,50, *$ \\
\hline \multirow[t]{7}{*}{ Brain } & M & 126 & & $1990 ; 0.26,0.007$ & $0.42, *$ & & & LE: $0.27, *$ & $0.71,101, *$ \\
\hline & & 130 & $0.16,0.036$ & $1990 ; 0.37, *$ & $0.38, *$ & & & & $0.68,91, *$ \\
\hline & & 145 & & & $0.59, *$ & & & LE: $0.35, *$ & $0.67,149, *$ \\
\hline & & 130 & & $1990 ; 0.44, *$ & $0.44, *$ & & & & $0.67,131, *$ \\
\hline & & 126 & & $1090 ; 0.58, *$ & & & & LE: $0.27,0.001$ & $0.64,111, *$ \\
\hline & $\mathrm{F}$ & 147 & $0.30, *$ & & $0.43, *$ & & & $\begin{array}{c}\text { Sweet93: } 0.18 \text {, } \\
0.006\end{array}$ & $0.54,59, *$ \\
\hline & & 148 & $0.36, *$ & & $0.47, *$ & & & & $0.53,83, *$ \\
\hline \multirow[t]{2}{*}{ Breast } & $\mathrm{F}$ & 142 & $0.22, *$ & $1993 ; 0.49, *$ & & $0.26,0.001$ & & & $0.76,147, *$ \\
\hline & & 142 & $0.28, *$ & & $0.17, *$ & $0.51, *$ & & & $0.70,111, *$ \\
\hline Cervix & $\mathrm{F}$ & 142 & & & $-0.40, *$ & $-0.30, *$ & & & $0.38,44, *$ \\
\hline \multirow[t]{4}{*}{ Colorectal } & M & 122 & $0.38, *$ & $1985 ; 0.21,0.013$ & & $0.25, *$ & & Alc93: $0.22, *$ & $0.84,160, *$ \\
\hline & & 138 & $0.35, *$ & & $0.17,0.007$ & $0.26, *$ & & Alc93: $0.31, *$ & $0.80,141, *$ \\
\hline & $\mathrm{F}$ & 131 & $0.36, *$ & $1985 ; 0.31, *$ & & & LE: $0.14,0.02$ & Alc93: 0.21, * & $0.77,112, *$ \\
\hline & & 136 & $0.35, *$ & $1985 ; 0.40, *$ & & & & Alc93: $0.23, *$ & $0.74,127, *$ \\
\hline Corpus uteri & $\mathrm{F}$ & 137 & $0.38, *$ & $1990 ; 0.37, *$ & & & & $\begin{array}{c}\text { Sweet90: } 0.21 \text {, } \\
0.001\end{array}$ & $0.68,97, *$ \\
\hline \multirow[t]{2}{*}{$\begin{array}{l}\text { Hodgkin's } \\
\text { lymphoma }\end{array}$} & M & 141 & & & $0.26,0.003$ & & & Fat00: 0.44, * & $0.41,49, *$ \\
\hline & $\mathrm{F}$ & 132 & & & $0.33, *$ & & & Fat $00: 0.36, *$ & $0.39,43, *$ \\
\hline
\end{tabular}


Table 3. Cont

\begin{tabular}{|c|c|c|c|c|c|c|c|}
\hline \multirow[t]{9}{*}{ Kidney } & $\mathrm{M}$ & 153 & $0.32, *$ & & $0.54, *$ & Cer93: $-0.26, *$ & $0.70,122, *$ \\
\hline & & 152 & $0.25, *$ & $1993 ; 0.39, *$ & $0.30, *$ & & $0.70,119, *$ \\
\hline & & 154 & $0.37, *$ & $1993 ; 0.53, *$ & & & $0.67,153, *$ \\
\hline & & 153 & $0.36, *$ & & $0.51, *$ & & $0.64,135, *$ \\
\hline & $\mathrm{F}$ & 153 & $0.24, *$ & $1993 ; 0,21,0.018$ & $0.45, *$ & $\begin{array}{c}\text { Cer93: }-0.15 \\
0.007\end{array}$ & $0.69,84, *$ \\
\hline & & 153 & $0.30, *$ & & $0.57, *$ & Cer93: $-0.21, *$ & $0.68,106, *$ \\
\hline & & 153 & $0.24, *$ & $1993 ; 0.34, *$ & $0.35, *$ & & $0.67,105, *$ \\
\hline & & 153 & $0.37, *$ & & $0.53, *$ & & $0.64,133, *$ \\
\hline & & 154 & $0.28, *$ & $1993 ; 0.57, *$ & & & $0.62,123, *$ \\
\hline \multirow[t]{2}{*}{ Laryngeal } & $\mathrm{M}$ & 157 & $0.68, *$ & & & & $0.46,131, *$ \\
\hline & $\mathrm{F}$ & 139 & $0.31, *$ & & & & $0.09,15, *$ \\
\hline \multirow[t]{3}{*}{ Leukemia } & $\mathrm{M}$ & 147 & $0.32, *$ & $2000 ; 0.38, *$ & & LE: $0.20,0.016$ & $0.62,78, *$ \\
\hline & $\mathrm{F}$ & 151 & $0.37, *$ & & & LE: $0.45, *$ & $0.52,81, *$ \\
\hline & & 146 & $0.32, *$ & $2000 ; 0.15,0.15$ & & LE: $0.36, *$ & $0.52,52, *$ \\
\hline \multirow[t]{2}{*}{ Lip, oral } & $\mathrm{M}$ & 157 & $0.45, *$ & & $-0.20,0.06$ & & $0.11,10, *$ \\
\hline & $\mathrm{F}$ & 156 & $0.29,0.002$ & & $-0.29,0.003$ & & $0.06,6,0.003$ \\
\hline \multirow[t]{2}{*}{ Liver } & $\mathrm{M}$ & 136 & & $1980 ;-0.37, *$ & & & $0.13,21, *$ \\
\hline & $\mathrm{F}$ & 136 & & $1980 ;-0.44, *$ & & & $0.19,33, *$ \\
\hline \multirow[t]{5}{*}{ Lung } & $\mathrm{M}$ & 68 & CigM; 0.62, * & & $0.33, *$ & & $0.68,72, *$ \\
\hline & & 68 & CigM; 0.63, * & & & AF00: $0.34, *$ & $0.67,68, *$ \\
\hline & & 68 & CigM; $0.60, *$ & & $0.23,0.04$ & AF00: $0.14,0.22$ & $0.68,49, *$ \\
\hline & $\mathrm{F}$ & 71 & CigF; 0.69,* & & & AF93; $0.26,0.001$ & $0.78,123, *$ \\
\hline & & 71 & CigF; 0.74,* & & $0.21,0.004$ & & $0.77,118, *$ \\
\hline
\end{tabular}


Table 3. Cont.

\begin{tabular}{|c|c|c|c|c|c|c|c|c|c|}
\hline \multirow[t]{4}{*}{$\begin{array}{l}\text { Multiple } \\
\text { myeloma }\end{array}$} & $\mathrm{M}$ & 112 & & $1985 ; 0.61, *$ & & $\begin{array}{l}0.22, \\
0.05\end{array}$ & & & $0.64,100, *$ \\
\hline & & 122 & & $1985 ; 0.66, *$ & & & & LE: $0.17,0.05$ & $0.61,97, *$ \\
\hline & $\mathrm{F}$ & 110 & & $1990 ; 0.33,0.003$ & & $0.49, *$ & & & $0.61,88, *$ \\
\hline & & 129 & & & & $0.55, *$ & & LE: $0.28,0.001$ & $0.60,85, *$ \\
\hline \multirow[t]{2}{*}{$\begin{array}{l}\text { Non-Hodgkin's } \\
\text { lymphoma }\end{array}$} & $\mathrm{M}$ & 142 & & & & $0.68, *$ & & & $0.46,120, *$ \\
\hline & $\mathrm{F}$ & 141 & & & & $0.71, *$ & & & $0.50,140, *$ \\
\hline \multirow[t]{2}{*}{ Ovarian } & $\mathrm{F}$ & 138 & $0.25,0.002$ & $1990 ; 0.55, *$ & & & & & $0.55,85, *$ \\
\hline & & & & & & & & Sweet90: & \\
\hline \multirow[t]{3}{*}{ Pancreatic } & $\mathrm{M}$ & 132 & $0.42, *$ & $1985 ; 0.28,0.001$ & & & Alc93: $0.18,0.005$ & $0.15,0.013$ & $0.74,93, *$ \\
\hline & & 132 & $0.45, *$ & $1990 ; 0.39, *$ & & & & $0.15,0.013$ & $0.73,116, *$ \\
\hline & $\mathrm{F}$ & 132 & $0.26,0.001$ & $1990 ; 0.46, *$ & & & & $0.20,0.003$ & $0.64,80, *$ \\
\hline \multirow[t]{3}{*}{ Prostate } & $\mathrm{M}$ & 137 & & $1980 ; 0.52, *$ & & & Cer93: $-0.23, *$ & Sweet90: $0.18,0.019$ & $0.56,58, *$ \\
\hline & & 132 & $0.45, *$ & $1990 ; 0.39, *$ & & & & $0.15,0.013$ & $0.73,116, *$ \\
\hline & $\mathrm{F}$ & 132 & $0.26,0.001$ & $1990 ; 0.46, *$ & & & & $0.20,0.003$ & $0.64,80, *$ \\
\hline Testicular & M & 123 & & $1985 ; 0.85, *$ & & & & & $0.72,312, *$ \\
\hline \multirow[t]{2}{*}{ Thyroid } & $\mathrm{M}$ & 146 & & $1993 ; 0.78, *$ & $-0.26,0.009$ & & & & $0.36,42, *$ \\
\hline & $\mathrm{F}$ & 152 & & $1993 ; 0.60, *$ & $-0.27,0.014$ & & & & $0.18,18, *$ \\
\hline
\end{tabular}

M: male; F: female; *: $p<0.001$; sweet90: sweetener supply, 1990; sweet93: sweetener supply, 1993. 
Table 4. Numbers of cancer types for which significant direct and inverse correlations were found.

\begin{tabular}{|c|c|c|c|c|c|c|}
\hline Factor & All Countries M & All Countries F & All Countries M/F & HQ Countries M & HQ Countries F & HQ Countries M/F \\
\hline \multicolumn{7}{|l|}{ Direct correlation } \\
\hline Alcohol & 1 & 1 & 1 & 1 & 1 & 1 \\
\hline Animal fat & 2 & 1 & 2 & 2 & 1 & 2 \\
\hline Animal product energy & 9 & 7 & 12 & 5 & 6 & 10 \\
\hline Fat & 1 & 1 & 1 & 1 & 1 & 1 \\
\hline GDP & 4 & 4 & 5 & 5 & 3 & 7 \\
\hline Latitude & 6 & 4 & 6 & 5 & 6 & 6 \\
\hline Life expectancy & 1 & 2 & 3 & 2 & 3 & 3 \\
\hline Lung cancer & 8 & 11 & 12 & 8 & 9 & 10 \\
\hline Sweeteners & 1 & 2 & 3 & 0 & 0 & 0 \\
\hline \multicolumn{7}{|l|}{ Inverse correlation } \\
\hline Animal product energy & 0 & 0 & 0 & 2 & 2 & 2 \\
\hline Cereals & 2 & 1 & 2 & 2 & 1 & 2 \\
\hline GDP & 0 & 1 & 1 & 0 & 1 & 1 \\
\hline Latitude & 2 & 3 & 3 & 0 & 2 & 2 \\
\hline
\end{tabular}

M: male; F: female; HQ: high quality countries. 
Meat consumption has been recognized as a risk factor for cancer since at least the early 20th century. A study of cancer rates in different ethnic groups in Chicago from 1900 to 1907 found that "heavy meat eaters - Germans, Irish, Scandinavians - apparently had high rates of cancer mortality, but pasta-consuming Italians and rice-eating Chinese had low rates. Though the survey was crude, it was more thorough than many of its kind (it studied some 4600 cases over a seven-year stretch from 1900 to 1907). Its conclusions concerning the potential risk of eating too much meat crudely anticipated those of many experts eighty years later" [36,37].

The present study as well as other ecological studies found dietary animal products associated with increased risk of chronic diseases. McCarty noted that several cancers increased in frequency in Asia, in southern Europe, and among African Americans in the 20th century after the increase in dietary animal products: breast, colon, kidney ovary, pancreas, and prostate [38]. A recent ecological study of trends of Alzheimer's disease rates in Japan correlated meat and animal products with a 15- to 25-year lag between dietary changes and prevalence of Alzheimer's disease [39]. Iron was also suggested as a link for that finding.

Mechanisms linking animal products to risk of cancer probably include increased production of insulin, insulin-like growth factor 1, and sex hormones: "Epidemiological evidence is accumulating and suggests that the risk of cancers of the colon, pancreas, endometrium, breast and prostate are related to circulating levels of insulin, (insulin-like growth factor 1), or both" [40]. Also, iron in meat may be a risk factor for cancer through increased production of free radicals and increased oxidative stress and DNA damage [41].

The correlation of energy from animal products with risk for a number of common cancers observed in this study may meet with some skepticism, inasmuch as, in prospective or case-control studies conducted in Western countries, such a correlation is rarely reported. Correlations with red meat or flesh foods cooked in high heat occasionally emerge (possibly reflecting an impact of heme iron, mutagens, or saturated fat), but animal products per se are rarely incriminated in such studies. A possible explanation may be that a moderate degree of essential amino acid restriction is required for down-regulation of IGF-I, mTORC1 activity, or other yet-unknown factors which act as cancer promoters $[42,43]$. Such restriction may be achievable with a vegan diet of moderate protein content, but diets that incorporate meaningful quantities of high-quality animal protein would presumably be too high in essential amino acids to down-regulate cancer promotional activities. And even vegans in Western countries often have an ample intake of isolated soy protein, which is of intermediate quality and capable of boosting IGF-I levels $[44,45]$. So only a tiny fraction of people in Western societies are likely to achieve a degree of essential amino acid restriction capable of modulating cancer risk-and most of these are only vegan for a portion of their life span. Varying protein intake within the range typical of Western societies may have little impact on cancer promotional activities.

\subsection{Latitude}

Several previous multicountry ecological studies interpreted findings of an index for annual solar UVB dose, which is strongly inversely correlated with latitude, as an index of vitamin D production. Such studies found inverse relations between that index and incidence rates for a number of types of cancer. Those cancers included bladder [46], brain [47], breast [48], colorectal [49], endometrial [50], 
kidney [51], lung [52], ovarian [53], pancreatic cancer [54], and leukemia [55]. However, under the Bonferroni principle ( $p$ must be $<0.05 / n$, where $n$ is the number of factors for significance to be claimed), the UVB index was significantly correlated with only bladder, brain, kidney, and lung cancer. This study confirmed these findings.

As to why this study did not find more cancers to have significant increases with increasing latitude, it appears that if animal products are significantly correlated with cancer incidence rates, it is less likely that latitude is. This seems to be the case for breast, corpus uteri, ovarian, and pancreatic cancer. For kidney cancer, when energy from animal products is included, the correlation for latitude is lower than when cereals are included.

In single-country studies, latitude is often a good index of solar UVB doses [56-58]. However, in the United States, considerable asymmetry exists in solar UVB doses owing to the geography [59]. However, the interpretation of latitude as an index of vitamin D production globally is questionable. The primary reason is that skin pigmentation varies with solar UV doses where people have lived for many generations $[60,61]$ so that serum 25-hydroxyvitamin $\mathrm{D}[25(\mathrm{OH}) \mathrm{D}]$ levels do not vary much globally. Three reviews found that country mean serum $25(\mathrm{OH}) \mathrm{D}$ levels are near $50 \mathrm{nmol} / \mathrm{L}$ in nearly every country [62-64]. The nearly constant serum 25(OH)D with respect to latitude is reasonable since if large differences existed, risk of vitamin D-sensitive diseases would vary considerably with respect to latitude. A pronounced increase of multiple sclerosis prevalence occurs with increasing latitude [65]. One study inversely correlated both solar UV dose and vitamin D with risk of multiple sclerosis [66].

The inverse correlation for cervical cancer can be understood in terms of higher risk of infections at low latitudes, and that for lip cancer in that UVB is a risk factor for lip cancer [22].

An alternative interpretation of latitude is that it may be an index of various modeled and/or unmodeled factors such as infection, living in urban regions, and eating processed food. For example, Toxoplasma gondii infection rates increase with latitude, and $T$. gondii is a risk factor for brain cancer [67].

\subsection{GDP}

Studies have often correlated per capita GDP with increased risk of chronic diseases, such as cancer [6] and diabetes [68]. Several possible reasons may account for why cancer rates are higher in countries with higher GDP: more time spent indoors, less time engaged in physical exercise [69], greater consumption of processed foods [70], greater life expectancy [71], more chemical pollution exposure, and reduced risk of infectious diseases [72,73].

\subsection{Life Expectancy}

Cancer risk does increase with life expectancy for most cancers in this study, such as breast and colorectal cancer (see Figure 1). However, life expectancy is significantly correlated with only three cancers: brain cancer, leukemia, and multiple myeloma. Why life expectancy is not significantly correlated with other cancers in this study is unclear. 
Figure 1. Breast cancer incidence rates for 2008 vs. life expectancy for males in 1990 with a third order fit.

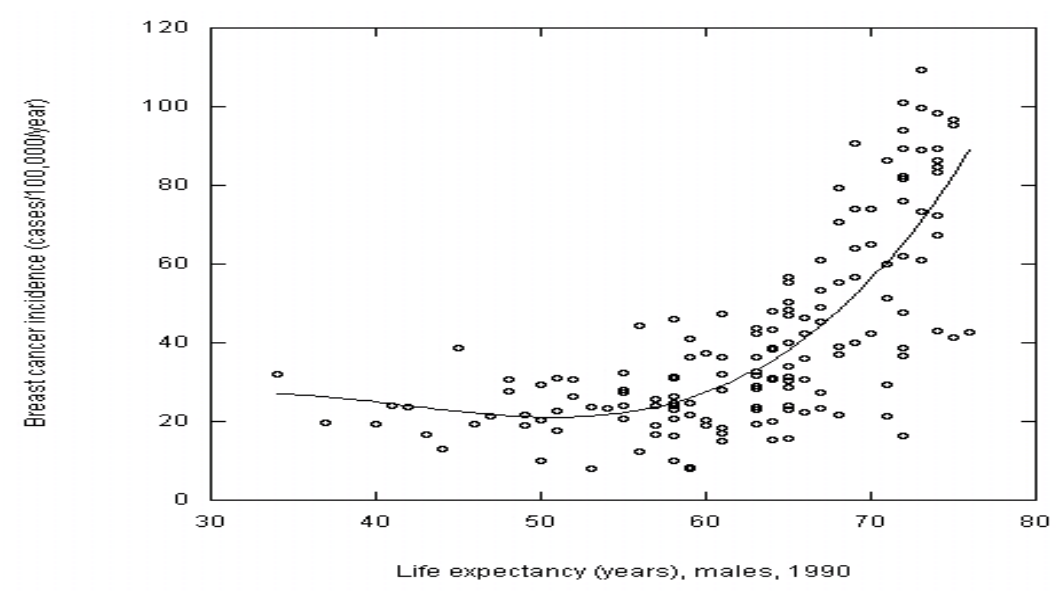

\subsection{Individual Cancers}

Table 5 lists journal papers supporting the findings in Tables 2 and 3. Findings from this ecological study generally agree with the journal literature for most factors for most cancers studied. Some results in the journal literature were not supported in this study, such as alcohol for several cancers, animal products for colorectal cancer, and latitude for many cancers. These failures indicate that such ecological studies can identify only up to three or four cancer risk-modifying factors as having significant correlations with cancer incidence rates in the best model for the particular cancer. Thus, findings by such ecological studies do not offer evidence that nonsignificant risk-modifying factors are not related to cancer risk, just that they seem less important than other factors.

This study found no significant results for esophageal, gallbladder, and stomach cancer. Esophageal and stomach cancer have infections as important risk factors (see Table 5).

Studies have inversely correlated white meat [74] and fish [75] with risk of liver cancer, whereas other studies directly correlated liver cancer risk with intake of red and processed meat $[74,75]$.

The directly correlation of latitude with melanoma risk is related to skin pigmentation. Melanoma incidence rates for people living in their ancestral homelands below $35^{\circ}$ latitude are very low (mean value, $\sim 2$ cases/100,000/year) but rise rapidly at higher latitudes (regression mean, $\sim 14$ for $>60^{\circ}$ latitude). The main reason for this latitudinal variation is variation in skin pigmentation, which has adjusted for protection against adverse effects of solar UV while permitting adequate production of vitamin D [56]. Australia and New Zealand have the highest melanoma incidence rates and are populated primarily by people who can trace their ancestry to northern Europe. GDP's direct correlation with melanoma incidence rates may be related to increased travel to sunny locations for those living in the wealthiest countries. Studies in Norway and the United States have correlated air travel with risk of melanoma [76,77]. 
Table 5. Journal literature supporting findings in this study.

\begin{tabular}{|c|c|c|c|c|c|c|c|}
\hline Cancer & Animal Products & Smoking & GDP per Capita & Latitude * & Alcohol & Misccellaneous & Infection \\
\hline Bladder & {$[78,79]$} & {$[80,81]$} & {$[82]$} & [46] UVB & & & \\
\hline Brain & [83] & [84] & & {$[47,67]$} & & & {$[67,85]$} \\
\hline Breast & {$[86,87]$} & & {$[82,88]$} & UVB & & & \\
\hline Cervix & & [80] & & & & & [89] \\
\hline Colorectal & {$[78,90,91]$} & {$[80]$} & {$[49,82,88]$} & UVB & {$[92,93]$} & Sugar [94] & [89] \\
\hline Corpus uteri & {$[78]$} & [95] & & uvb & & Sugar [96] & \\
\hline Esophageal & {$[90,91,97]$} & {$[80,81]$} & & uvb & [92] & & [98] \\
\hline Gallbladder & & & & uvb & & & \\
\hline Gastric & & [81] & & uvb & & & [99] \\
\hline $\begin{array}{l}\text { Hodgkin's } \\
\text { lymphoma }\end{array}$ & [100] & & & uvb & & & [101] \\
\hline Kidney & [79] & [102] & [82] & $\begin{array}{c}{[51,103]} \\
\text { UVB }\end{array}$ & & Cereals $[104,105]$ & \\
\hline Laryngeal & & {$[80,81]$} & & uvb & [92] & & [106] \\
\hline Leukemia & [107] & {$[107]$} & & [55] & & & \\
\hline Lip & & [108] & & [108] (direct) & & & \\
\hline Liver & {$[74,90]$} & {$[81,102]$} & & & & & [109] \\
\hline Lung & {$[87,91,110]$} & [81] & [88] & [52] uvb & & & \\
\hline Mult. Myel. & {$[100,111]$} & & & & & & [112] \\
\hline $\begin{array}{l}\text { Non-Hodgkin's } \\
\text { lymphoma }\end{array}$ & {$[113,114]$} & & {$[82,88]$} & UVB & & & [115] \\
\hline Ovarian & {$[78,116,117]$} & [118] & [82] & [53] UVB & & & \\
\hline Pancreatic & {$[78,87,119]$} & {$[80,81]$} & [88] & [54] UVB & {$[92,120]$} & Sugar [94] & \\
\hline Prostate & [79] & [121] (inverse) & {$[82,88]$} & uvb & [122] & Sugar [94], rice [121] & \\
\hline Testicular & [79] & & & & & Fat [123] & \\
\hline Thyroid & [124] & & & uvb & & & \\
\hline
\end{tabular}

UVB: strong support for UVB from single-country studies [9,56-58,125]; uvb: moderate support. 


\subsection{Strengths and Limitations of This Study}

Ecological studies such as those reported here have several strengths and limitations. Strengths include that such ecological studies involve many cases, permitting investigation of many cancers and consideration of many factors. Also, since data exist for several indices of cancer risk-modifying factors over many years, ecological studies can investigate the effect of delay between risk-modifying factor and cancer incidence.

Limitations include that the data are for country populations as a whole, so values for risk-modifying factors may not accurately reflect risk factors on individuals who develop cancer. For example, solar UVB doses vary widely in mid-latitude countries with large latitude ranges. Also, dietary intake varies widely by individual. Further, dietary supply data are based on food that enters the consumer supply and does not account for waste, loss, and spoilage. This study assumes that consumption rates are a similar fraction of consumer supply for each country, probably around $70 \%$. Also, country-averaged data for some cancer risk-modifying factors, such as rates for obesity, physical exercise, and diseases that may compete with risk of cancer (e.g., cardiovascular disease and infectious diseases), are either not available or were not included in the analysis. In addition, there are cancer risk-modifying factors that are particular to a few countries or regions within countries. Examples of such factors include poor sewage disposal, which is a risk for Helicobacter pylori infection and gastric cancer [126], arsenic in drinking water and risk of renal cancer [127], industrial pollution, a risk factor for several types of cancer [128], betel quid chewing and risk of head and neck cancers [129].

\section{Conclusions}

This multicountry ecological study of cancer incidence rates in 2008 found that lung cancer incidence rates, supply of energy derived from animal products, latitude (an index for solar UVB doses), and per capita GDP had the largest number of significant correlations with various cancers. Alcoholic beverages, animal fat, life expectancy, and sweeteners had significant correlations with a few cancers. This study found inverse correlations for cereals, per capita GDP, and latitude for one to three cancers each. For all countries, the models explained $9 \%-84 \%$ of the variance, with median values of $62 \%$ for males and $54.5 \%$ for females. For countries with high-quality data, the models explained $6 \%-75 \%$ of the variance, with median values of $55 \%$ for males and $41.5 \%$ for females. Since most factors considered are environmental, rather than genetic, one may reasonably conclude that environmental causes of cancer contribute much more to cancer risk than do genetic factors, and thus lifestyle changes could reduce much of the cancer burden.

\section{Conflicts of Interest}

The authors declare no conflict of interest.

\section{References}

1. Ferlay, J.; Shin, H.R.; Bray, F.; Forman, D.; Mathers, C.; Parkin, D.M. GLOBOCAN 2008, Cancer Incidence and Mortality Worldwide: IARC CancerBase No. 10. Int. J. Cancer 2010, 127. Available online: http://globocan.iarc.fr/factsheet.asp (accessed on 29 August 2013). 
2. Siegel, R.; Ward, E.; Brawley, O.; Jemal, A. Cancer statistics, 2011: The impact of eliminating socioeconomic and racial disparities on premature cancer deaths. CA Cancer J. Clin. 2011, 61, 212-236.

3. Hoyert, D.L.; Xu, J. Deaths: Preliminary data for 2011. Nat. Vital Stat. Rep. 2012, 61, 8.

4. Cancer. Available online: http://www.who.int/mediacentre/factsheets/fs297/en/ (accessed on 4 November 2013).

5. The American Institute for Cancer Research/World Cancer Research Fund. Policy and Action for Cancer Prevention. Food, Nutrition and Physical Activity: A Global Perspective; AICR: Washington, DC, USA, 2009.

6. Armstrong, B.; Doll, R. Environmental factors and cancer incidence and mortality in different countries, with special reference to dietary practices. Int. J. Cancer 1975, 15, 617-631.

7. Garland, C.F.; Garland, F.C. Do sunlight and vitamin D reduce the likelihood of colon cancer? Int. J. Epidemiol. 1980, 9, 227-231.

8. Garland, C.F.; Garland, F.C.; Gorham, E.D.; Lipkin, M.; Newmark, H.; Mohr, S.B.; Holick, M.F. The role of vitamin D in cancer prevention. Am. J. Public Health 2006, 96, 252-261.

9. Grant, W.B.; Garland, C.F. The association of solar ultraviolet B (UVB) with reducing risk of cancer: Multifactorial ecologic analysis of geographic variation in age-adjusted cancer mortality rates. Anticancer Res. 2006, 26, 2687-2699.

10. Grant, W.B. Ecological studies of the UVB-Vitamin D-Cancer hypothesis. Anticancer Res. 2012, 32, 223-236.

11. Garland, C.F.; Gorham, E.D.; Mohr, S.B.; Garland, F.C. Vitamin D for cancer prevention: Global perspective. Ann. Epidemiol. 2009, 19, 468-483.

12. Moukayed, M.; Grant, W.B. Molecular link between vitamin D and cancer prevention. Nutrients 2013, 5, 3993-4021.

13. Hill, A.B. The environment and disease: Association or causation? Proc. Royal Soc. Med. 1965, 58, 295-300.

14. Grant, W.B. How strong is the evidence that solar ultraviolet B and vitamin D reduce the risk of cancer? An examination using Hill's criteria for causality. Dermato-Endocrinology 2009, 1, 17-24.

15. Mohr, S.B.; Gorham, E.D.; Alcaraz, J.E.; Kane, C.I.; Macera, C.A.; Parsons, J.K.; Wingard, D.L.; Garland, C.F. Does the evidence for an inverse relationship between serum vitamin D status and breast cancer risk satisfy the Hill criteria? Dermato-Endocrinology 2012, 4, 152-157.

16. Food and Agriculture Organization of the United Nations. Food Balance Sheets, 1992-94 Average. FAO: Rome, Italy, 1997.

17. Harashima, E.; Nakagawa, Y.; Urata, G.; Tsuji, K.; Shirataka, M.; Matsumura, Y. Time-lag estimate between dietary intake and breast cancer mortality in Japan. Asia Pac. J. Clin. Nutr. 2007, 16, 193-198.

18. Zhang, J.; Dhakal, I.B.; Zhao, Z.; Li, L. Trends in mortality from cancers of the breast, colon, prostate, esophagus, and stomach in East Asia: Role of nutrition transition. Eur. J. Cancer Prev. 2012, 21, 480-489.

19. World Health Organization. Tables of Causes of Death and Disability Adjusted Life Years by Country 2004. Available online: http://www.who.int/healthinfo/statistics/bodgbddeathdalyesti mates.xls (accessed on 5 November 2013). 
20. Mathers, C.D.; Bernard, C.; Iburg, K.; Inoue, M.; Ma Fat, D.; Shibuya, K.; Stein, C.; Tomijima, N. The Global Burden of Disease in 2002: Data Sources, Methods and Results. In GPE Discussion Paper No. 54; World Health Organization: Geneva, Swizerland, 2003.

21. Food and Agriculture Organization of the United Nations. Food Balance Sheets. Available online: http://faostat.fao.org/site/368/default.aspx\#ancor (accessed on 4 November 2013).

22. Grant, W.B. Role of solar UVB irradiance and smoking in cancer as inferred from cancer incidence rates by occupation in Nordic countries. Dermato-Endocrinology 2012, 4, 203-211.

23. Grant, W.B. An ecologic study of cancer mortality rates in Spain with respect to indices of solar UVB irradiance and smoking. Int. J. Cancer 2007, 120, 1123-1128.

24. Bilal, U.; Fernandez, E.; Beltran, P.; Navas-Acien, A.; Bolumar, F.; Franco, M. Validation of a method for reconstructing historical rates of smoking prevalence. Am. J. Epidemiol. 2013, doi: 10.1093/aje/kwt224.

25. Afzal, S.; Bojesen, S.E.; Nordestgaard, B.G. Low plasma 25-hydroxyvitamin D and risk of tobacco-related cancer. Clin. Chem. 2013, 59, 771-780.

26. Data on Tobacco Consumption. Available online: http://www.who.int/tobacco/media/en/Poland.pdf (accessed on 20 December 2013).

27. International Monetary Fund. List of Countries by Past and Future GDP (PPP) per Capita. Available online: http://en.wikipedia.org/w/index.php?title=List_of_countries_by_pastand_futur _GDP_(PPP)_per_capita\&oldid=574426146 (accessed on 4 November 2013).

28. Central Intelligence Agency. The CIA World Fact Book 2008; Central Intelligence Agency: Washington, DC, USA, 2008.

29. KaleidaGraph, version 4.2; Synergy Software: Reading, PA, USA, 2005.

30. Tsuji, K.; Harashima, E.; Nakagawa, Y.; Urata, G.; Shirataka, M. Time-lag effect of dietary fiber and fat intake ratio on Japanese colon cancer mortality. Biomed. Environ. Sci. 1996, 9, 223-228.

31. Andersson, S.O.; Baron, J.; Wolk, A.; Lindgren, C.; Bergström, R.; Adami, H.O. Early life risk factors for prostate cancer: A population-based case-control study in Sweden. Cancer Epidemiol. Biomark. Prev. 1995, 4, 187-192.

32. John, E.M.; Koo, J.; Schwartz, G.G. Sun exposure and prostate cancer risk: Evidence for a protective effect of early-life exposure. Cancer Epidemiol. Biomark. Prev. 2007, 16, 1283-1286.

33. Hirayama, T. Mortality in Japanese with life-styles similar to Seventh-Day Adventists: Strategy for risk reduction by life-style modification. Nat. Cancer Inst. Monogr. 1985, 69, 143-153.

34. Fraser, G.E. Associations between diet and cancer, ischemic heart disease, and all-cause mortality in non-Hispanic white California Seventh-day Adventists. Am. J. Clin. Nutr. 1999, 70, 532S-538S.

35. Thygesen, L.C.; Hvidt, N.C.; Hansen, H.P.; Hoff, A.; Ross, L.; Johansen, C. Cancer incidence among Danish Seventh-day Adventists and Baptists. Cancer Epidemiol. 2012, 36, 513-518.

36. Cancer Increasing among Meat Eaters. New York Times, Sep 24, 1907. Available online: http://query.nytimes.com/gst/abstract.html?res=9502E1D81331E733A25757C2A96F9C946697 D6CF (accessed on 24 December 2013).

37. Patterson, J.T. The Dread Disease; Cancer and Modern Culture; Harvard University Press: Cambridge, MA, USA, 1987; pp. 44-45. 
38. McCarty, M.F. Mortality from Western cancers rose dramatically among African-Americans during the 20th century: Are dietary animal products to blame? Med. Hypotheses 2001, 57, 169-174.

39. Grant, W.B. Trends in diet and Alzheimer's disease during the nutrition transition in Japan and developing countries. J. Alzheimer's Dis. 2013, 38, 611-620.

40. Kaaks, R. Nutrition, insulin, IGF-1 metabolism and cancer risk: A summary of epidemiological evidence. Novartis Found. Symp. 2004, 262, 247-260; discussion 260-268.

41. Grant, W.B. An ecological study of cancer mortality rates including indices for dietary iron and zinc. Anticancer Res. 2008, 28, 1955-1963.

42. McCarty, M.F. Insulin and IGF-I as determinants of low "Western" cancer rates in the rural third world. Int. J. Epidemiol. 2004, 33, 908-910.

43. Fontana, L.; Weiss, E.P.; Villareal, D.T.; Klein, S.; Holloszy, J.O. Long-term effects of calorie or protein restriction on serum IGF-1 and IGFBP-3 concentration in humans. Aging Cell 2008, 7, 681-687.

44. Allen, N.E.; Appleby, P.N.; Davey, G.K.; Kaaks, R.; Rinaldi, S.; Key, T.J. The associations of diet with serum insulin-like growth factor I and its main binding proteins in 292 women meat-eaters, vegetarians, and vegans. Cancer Epidemiol. Biomark. Prev. 2002, 11, 1441-1448.

45. Dewell, A.; Weidner, G.; Sumner, M.D.; Barnard, R.J.; Marlin, R.O.; Daubenmier, J.J.; Chi, C.; Carroll, P.R.; Ornish, D. Relationship of dietary protein and soy isoflavones to serum IGF-1 and IGF binding proteins in the Prostate Cancer Lifestyle Trial. Nutr. Cancer 2007, 58, 35-42.

46. Mohr, S.B.; Garland, C.F.; Gorham, E.D.; Grant, W.B.; Garland, F.C. Ultraviolet B irradiance and incidence rates of bladder cancer in 174 countries. Am. J. Prev. Med. 2010, 38, 296-302.

47. Mohr, S.B.; Gorham, E.D.; Garland, C.F.; Grant, W.B.; Garland, F.C. Low ultraviolet B and increased risk of brain cancer: an ecological study of 175 countries. Neuroepidemiology 2010, 35, 281-290.

48. Mohr, S.B.; Garland, C.F.; Gorham, E.D.; Grant, W.B.; Garland, F.C. Relationship between low ultraviolet B irradiance and higher breast cancer risk in 107 countries. Breast J. 2008, 14, 255-260.

49. Cuomo, R.; Mohr, S.B.; Gorham, E.D.; Garland, C.F. What is the relationship between ultraviolet B and global incidence rates of colorectal cancer? Dermato-Endocrinology 2013, 5, 181-185.

50. Mohr, S.B.; Garland, C.F.; Gorham, E.D.; Grant, W.B.; Garland, F.C. Is ultraviolet B irradiance inversely associated with incidence rates of endometrial cancer: An ecological study of 107 countries. Prev. Med. 2007, 45, 327-331.

51. Mohr, S.B.; Gorham, E.D.; Garland, C.F.; Grant, W.B.; Garland, F.C. Are low ultraviolet B and high animal protein intake associated with risk of renal cancer? Int. J. Cancer 2006, 119, 2705-2709.

52. Mohr, S.B.; Garland, C.F.; Gorham, E.D.; Grant, W.B.; Garland, F.C. Could ultraviolet B irradiance and vitamin D be associated with lower incidence rates of lung cancer? J. Epidemiol. Community Health 2008, 62, 69-74.

53. Garland, C.F.; Mohr, S.B.; Gorham, E.D.; Grant, W.B.; Garland, F.C. Role of ultraviolet B irradiance and vitamin D in prevention of ovarian cancer. Am. J. Prev. Med. 2006, 31, 512-514. 
54. Mohr, S.B.; Garland, C.F.; Gorham, E.D.; Grant, W.B.; Garland, F.C. Ultraviolet B irradiance and vitamin D status are inversely associated with incidence rates of pancreatic cancer worldwide. Pancreas 2010, 39, 669-674.

55. Mohr, S.B.; Garland, C.F.; Gorham, E.D.; Grant, W.B.; Garland, F.C. Ultraviolet B and incidence rates of leukemia worldwide. Am. J. Prev. Med. 2011, 41, 68-74.

56. Mizoue, T. Ecological study of solar radiation and cancer mortality in Japan. Health Phys. 2004, $87,532-538$.

57. Astbury, A. Non Uniformity in Cancer Mortality in the USA and Australia Appears to Share a Common Pathway. Triumf Report, TRI-PP-05-01, 2005. Available online: http://www.triumf.ca/ publications/pub/arch05/pp-05-01.pdf (accessed on 21 November 2011).

58. Chen, W.; Clements, M.; Rahman, B.; Zhang, S.; Qiao, Y.; Armstrong, B.K. Relationship between cancer mortality/incidence and ambient ultraviolet B irradiance in China. Cancer Causes Control 2010, 21, 1701-1709.

59. Fioletov, V.E.; McArthur, L.J.; Mathews, T.W.; Marrett, L. Estimated ultraviolet exposure levels for a sufficient vitamin D status in North America. J. Photochem. Photobiol. 2010, 100, 57-66.

60. Jablonski, N.G.; Chaplin, G. The evolution of human skin coloration. J. Hum. Evol. 2000, 39, 57-106.

61. Jablonski, N.G.; Chaplin, G. Colloquium paper: Human skin pigmentation as an adaptation to UV radiation. Proc. Natl. Acad. Sci. USA 2010, 107, 8962-8968.

62. Hagenau, T.; Vest, R.; Gissel, T.N.; Poulsen, C.S.; Erlandsen, M.; Mosekilde, L.; Vestergaard, P. Global vitamin D levels in relation to age, gender, skin pigmentation and latitude: An ecologic meta-regression analysis. Osteoporos Int. 2009, 20, 133-140.

63. Van Schoor, N.M.; Lips, P. Worldwide vitamin D status. Best Pract. Res. Clin. Endocrinol. Metab. 2011, 25, 671-680.

64. Hilger, J.; Friedel, A.; Herr, R.; Rausch, T.; Roos, F.; Wahl, D.A.; Pierroz, D.D.; Weber, P.; Hoffmann, K. A systematic review of vitamin D status in populations worldwide. Br. J. Nutr. 2013, doi:10.1017/S0007114513001840.

65. Kurtzke, J.F. Epidemiologic evidence for multiple sclerosis as an infection. Clin. Microbiol. Rev. 1993, 6, 382-427.

66. Lucas, R.M.; Ponsonby, A.L.; Dear, K.; Valery, P.C.; Pender, M.P.; Taylor, B.V.; Kilpatrick, T.J.; Dwyer, T.; Coulthard, A.; Chapman, C.; et al. Sun exposure and vitamin D are independent risk factors for CNS demyelination. Neurology 2011, 76, 540-548.

67. Thomas, F.; Lafferty, K.D.; Brodeur, J.; Elguero, E.; Gauthier-Clerc, M.; Misse, D. Incidence of adult brain cancers is higher in countries where the protozoan parasite Toxoplasma gondii is common. Biol. Lett. 2012, 8, 101-103.

68. Basu, S.; Yoffe, P.; Hills, N.; Lustig, R.H. The relationship of sugar to population-level diabetes prevalence: An econometric analysis of repeated cross-sectional data. PLoS One 2013, 8, e57873.

69. Bosdriesz, J.R.; Witvliet, M.I.; Visscher, T.L.; Kunst, A.E. The influence of the macro-environment on physical activity: A multilevel analysis of 38 countries worldwide. Int. J. Behav. Nutr. Phys. Act. 2012, 9, 110.

70. Hawkes, C. The role of foreign direct investment in the nutrition transition. Public Health Nutr. 2005, 8, 357-365. 
71. Renton, A.; Wall, M.; Lintott, J. Economic growth and decline in mortality in developing countries: An analysis of the World Bank development datasets. Public Health 2012, 126, 551-560.

72. Huicho, L.; Trelles, M.; Gonzales, F.; Mendoza, W.; Miranda, J. Mortality profiles in a country facing epidemiological transition: An analysis of registered data. BMC Public Health 2009, 9, 47.

73. Shuman, E.K. Global climate change and infectious diseases. Int. J. Occup. Environ. Med. 2011, 2, 11-19.

74. Freedman, N.D.; Cross, A.J.; McGlynn, K.A.; Abnet, C.C.; Park, Y.; Hollenbeck, A.R.; Schatzkin, A.; Everhart, J.E.; Sinha, R. Association of meat and fat intake with liver disease and hepatocellular carcinoma in the NIH-AARP cohort. J. Natl. Cancer Inst. 2010, 102, 1354-1365.

75. Fedirko, V.; Trichopolou, A.; Bamia, C.; Duarte-Salles, T.; Trepo, E.; Aleksandrova, K.; Nothlings, U.; Lukanova, A.; Lagiou, P.; Boffetta, P.; et al. Consumption of fish and meats and risk of hepatocellular carcinoma: The European Prospective Investigation into Cancer and Nutrition (EPIC). Ann. Oncol. 2013, 24, 2166-2173.

76. Bentham, G.; Aase, A. Incidence of malignant melanoma of the skin in Norway, 1955-1989: associations with solar ultraviolet radiation, income and holidays abroad. Int. J. Epidemiol. 1996, $25,1132-1138$.

77. Agredano, Y.Z.; Chan, J.L.; Kimball, R.C.; Kimball, A.B. Accessibility to air travel correlates strongly with increasing melanoma incidence. Melanoma Res. 2006, 16, 77-81.

78. Tavani, A.; La Vecchia, C.; Gallus, S.; Lagiou, P.; Trichopoulos, D.; Levi, F.; Negri, E. Red meat intake and cancer risk: A study in Italy. Int. J. Cancer 2000, 86, 425-428.

79. Oplander, C.; Volkmar, C.M.; Paunel-Gorgulu, A.; van Faassen, E.E.; Heiss, C.; Kelm, M.; Halmer, D.; Murtz, M.; Pallua, N.; Suschek, C.V. Whole body UVA irradiation lowers systemic blood pressure by release of nitric oxide from intracutaneous photolabile nitric oxide derivates. Circ. Res. 2009, 105, 1031-1040.

80. Thun, M.J.; Henley, S.J.; Calle, E.E. Tobacco use and cancer: An epidemiologic perspective for geneticists. Oncogene 2002, 21, 7307-7325.

81. Yun, Y.H.; Jung, K.W.; Bae, J.M.; Lee, J.S.; Shin, S.A.; Min Park, S.; Yoo, T.; Yul Huh, B. Cigarette smoking and cancer incidence risk in adult men: National Health Insurance Corporation Study. Cancer Detect. Prev. 2005, 29, 15-24.

82. Bray, F.; Jemal, A.; Grey, N.; Ferlay, J.; Forman, D. Global cancer transitions according to the Human Development Index (2008-2030): A population-based study. Lancet Oncol. 2012, 13, 790-801.

83. Kaplan, S.; Novikov, I.; Modan, B. Nutritional factors in the etiology of brain tumors: Potential role of nitrosamines, fat, and cholesterol. Am. J. Epidemiol. 1997, 146, 832-841.

84. Mandelzweig, L.; Novikov, I.; Sadetzki, S. Smoking and risk of glioma: A meta-analysis. Cancer Causes Control 2009, 20, 1927-1938.

85. Alibek, K.; Kakpenova, A.; Baiken, Y. Role of infectious agents in the carcinogenesis of brain and head and neck cancers. Infect. Agents Cancer 2013, 8, 7.

86. Ishimoto, H.; Nakamura, H.; Miyoshi, T. Epidemiological study on relationship between breast cancer mortality and dietary factors. Tokushima J. Exp. Med. 1994, 41, 103-114. 
87. Hu, J.; La Vecchia, C.; DesMeules, M.; Negri, E.; Mery, L. Meat and fish consumption and cancer in Canada. Nutr. Cancer 2008, 60, 313-324.

88. Knaul, F.M.; Adami, H.O.; Adebamowo, C.; Arreola-Ornelas, H.; Berger, A.J.; Bhadelia, A.; Cleary, J.; Hunter, D.J.; Keating, N.; Mebewu, A.; et al. The Global Cancer Divide: An Equity Imperative. In Closing the Cancer Divide: An Equity Imperative; Harvard University: Cambridge, MA, USA, 2012.

89. Zur Hausen, H. Papillomaviruses in the causation of human cancers-A brief historical account. Virology 2009, 384, 260-265.

90. Cross, A.J.; Leitzmann, M.F.; Gail, M.H.; Hollenbeck, A.R.; Schatzkin, A.; Sinha, R. A prospective study of red and processed meat intake in relation to cancer risk. PLoS Med. 2007, 4, e325.

91. Aune, D.; de Stefani, E.; Ronco, A.; Boffetta, P.; Deneo-Pellegrini, H.; Acosta, G.; Mendilaharsu, M. Meat consumption and cancer risk: A case-control study in Uruguay. Asian Pac. J. Cancer Prev. 2009, 10, 429-436.

92. Haas, S.L.; Ye, W.; Lohr, J.M. Alcohol consumption and digestive tract cancer. Curr. Opin. Clin. Nutr. Metab. Care 2012, 15, 457-467.

93. Nan, H.; Lee, J.E.; Rimm, E.B.; Fuchs, C.S.; Giovannucci, E.L.; Cho, E. Prospective study of alcohol consumption and the risk of colorectal cancer before and after folic acid fortification in the United States. Ann. Epidemiol. 2013, 23, 558-563.

94. Hu, J.; La Vecchia, C.; Augustin, L.S.; Negri, E.; de Groh, M.; Morrison, H.; Mery, L.; Canadian Cancer Registries Epidemiology Research Group. Glycemic index, glycemic load and cancer risk. Ann. Oncol. 2013, 24, 245-251.

95. Al-Zoughool, M.; Dossus, L.; Kaaks, R.; Clavel-Chapelon, F.; Tjonneland, A.; Olsen, A.; Overvad, K.; Boutron-Ruault, M.C.; Gauthier, E.; Linseisen, J.; et al. Risk of endometrial cancer in relationship to cigarette smoking: Results from the EPIC study. Int. J. Cancer 2007, 121, 2741-2747.

96. Zemla, B.; Guminski, S.; Franek, K.; Kolosza, Z.; Banasik, R. Etiological factors in invasive corpus uteri carcinoma. Neoplasma 1991, 38, 157-163.

97. Choi, Y.; Song, S.; Song, Y.; Lee, J.E. Consumption of red and processed meat and esophageal cancer risk: Meta-analysis. World J. Gastroenterol. 2013, 19, 1020-1029.

98. Zheng, S.; Vuitton, L.; Sheyhidin, I.; Vuitton, D.A.; Zhang, Y.; Lu, X. Northwestern China: A place to learn more on oesophageal cancer. Part one: Behavioural and environmental risk factors. Eur. J. Gastroenterol. Hepatol. 2010, 22, 917-925.

99. Crew, K.D.; Neugut, A.I. Epidemiology of gastric cancer. World J. Gastroenterol. 2006, 12, 354-362.

100. Tavani, A.; Pregnolato, A.; Negri, E.; Franceschi, S.; Serraino, D.; Carbone, A.; La Vecchia, C. Diet and risk of lymphoid neoplasms and soft tissue sarcomas. Nutr. Cancer 1997, 27, 256-260.

101. Hjalgrim, H. On the aetiology of Hodgkin lymphoma. Dan. Med. J. 2012, 59, B4485.

102. Ray, G.; Henson, D.E.; Schwartz, A.M. Cigarette smoking as a cause of cancers other than lung cancer: An exploratory study using the Surveillance, Epidemiology, and End Results Program. Chest 2010, 138, 491-499. 
103. Karami, S.; Boffetta, P.; Stewart, P.; Rothman, N.; Hunting, K.L.; Dosemeci, M.; Berndt, S.I.; Brennan, P.; Chow, W.H.; Moore, L.E. Occupational sunlight exposure and risk of renal cell carcinoma. Cancer 2010, 116, 2001-2010.

104. La Vecchia, C.; Chatenoud, L.; Negri, E.; Franceschi, S. Session: Whole cereal grains, fibre and human cancer wholegrain cereals and cancer in Italy. Proc. Nutr. Soc. 2003, 62, 45-49.

105. Daniel, C.R.; Park, Y.; Chow, W.H.; Graubard, B.I.; Hollenbeck, A.R.; Sinha, R. Intake of fiber and fiber-rich plant foods is associated with a lower risk of renal cell carcinoma in a large US cohort. Am. J. Clin. Nutr. 2013, 97, 1036-1043.

106. Li, X.; Gao, L.; Li, H.; Gao, J.; Yang, Y.; Zhou, F.; Gao, C.; Li, M.; Jin, Q. Human papillomavirus infection and laryngeal cancer risk: A systematic review and meta-analysis. J. Infect. Dis. 2013, 207, 479-488.

107. Ma, X.; Park, Y.; Mayne, S.T.; Wang, R.; Sinha, R.; Hollenbeck, A.R.; Schatzkin, A.; Cross, A.J. Diet, lifestyle, and acute myeloid leukemia in the NIH-AARP cohort. Am. J. Epidemiol. 2010, 171, 312-322.

108. Maruccia, M.; Onesti, M.G.; Parisi, P.; Cigna, E.; Troccola, A.; Scuderi, N. Lip cancer: A 10-year retrospective epidemiological study. Anticancer Res. 2012, 32, 1543-1546.

109. Ringelhan, M.; Heikenwalder, M.; Protzer, U. Direct effects of hepatitis B virus-encoded proteins and chronic infection in liver cancer development. Dig. Dis. 2013, 31, 138-151.

110. Kubik, A.; Zatloukal, P.; Tomasek, L.; Dolezal, J.; Syllabova, L.; Kara, J.; Kopecky, P.; Plesko, I. A case-control study of lifestyle and lung cancer associations by histological types. Neoplasma 2008, 55, 192-199.

111. Vlajinac, H.D.; Pekmezovic, T.D.; Adanja, B.J.; Marinkovic, J.M.; Kanazir, M.S.; Suvajdzic, N.D.; Colovic, M.D. Case-control study of multiple myeloma with special reference to diet as risk factor. Neoplasma 2003, 50, 79-83.

112. McShane, C.M.; Murray, L.J.; Engels, E.A.; Landgren, O.; Anderson, L.A. Common community-acquired infections and subsequent risk of multiple myeloma: A population-based study. Int. J. Cancer 2013, doi:10.1002/ijc. 28479.

113. Zheng, T.; Holford, T.R.; Leaderer, B.; Zhang, Y.; Zahm, S.H.; Flynn, S.; Tallini, G.; Zhang, B.; Zhou, K.; Owens, P.H.; et al. Diet and nutrient intakes and risk of non-Hodgkin's lymphoma in Connecticut women. Am. J. Epidemiol. 2004, 159, 454-466.

114. Van Leeuwen, M.T.; Turner, J.J.; Falster, M.O.; Meagher, N.S.; Joske, D.J.; Grulich, A.E.; Giles, G.G.; Vajdic, C.M. Latitude gradients for lymphoid neoplasm subtypes in Australia support an association with ultraviolet radiation exposure. Int. J. Cancer 2013, 133, 944-951.

115. Liu, Y.C.; Yang, Y.H.; Hsiao, H.H.; Yang, W.C.; Liu, T.C.; Chang, C.S.; Yang, M.Y.; Lin, P.M.; Hsu, J.F.; Chang, P.Y.; et al. Herpes zoster is associated with an increased risk of subsequent lymphoid malignancies - A nationwide population-based matched-control study in Taiwan. BMC Cancer 2012, 12, 503.

116. Risch, H.A.; Jain, M.; Marrett, L.D.; Howe, G.R. Dietary fat intake and risk of epithelial ovarian cancer. J. Natl. Cancer Inst. 1994, 86, 1409-1415.

117. Kolahdooz, F.; Ibiebele, T.I.; van der Pols, J.C.; Webb, P.M. Dietary patterns and ovarian cancer risk. Am. J. Clin. Nutr. 2009, 89, 297-304. 
118. Beral, V.; Gaitskell, K.; Hermon, C.; Moser, K.; Reeves, G.; Peto, R. Ovarian cancer and smoking: Individual participant meta-analysis including 28,114 women with ovarian cancer from 51 epidemiological studies. Lancet Oncol. 2012, 13, 946-956.

119. Ghadirian, P.; Thouez, J.P.; PetitClerc, C. International comparisons of nutrition and mortality from pancreatic cancer. Cancer Detect. Prev. 1991, 15, 357-362.

120. Anderson, M.A.; Zolotarevsky, E.; Cooper, K.L.; Sherman, S.; Shats, O.; Whitcomb, D.C.; Lynch, H.T.; Ghiorzo, P.; Rubinstein, W.S.; Vogel, K.J.; et al. Alcohol and tobacco lower the age of presentation in sporadic pancreatic cancer in a dose-dependent manner: A multicenter study. Am. J. Gastroenterol. 2012, 107, 1730-1739.

121. Severson, R.K.; Nomura, A.M.; Grove, J.S.; Stemmermann, G.N. A prospective study of demographics, diet, and prostate cancer among men of Japanese ancestry in Hawaii. Cancer Res. 1989, 49, 1857-1860.

122. Sawada, N.; Inoue, M.; Iwasaki, M.; Sasazuki, S.; Yamaji, T.; Shimazu, T.; Tsugane, S. Alcohol and smoking and subsequent risk of prostate cancer in Japanese men: The Japan Public Health Center-Based prospective study. Int. J. Cancer 2014, 134, 971-978.

123. Sigurdson, A.J.; Chang, S.; Annegers, J.F.; Duphorne, C.M.; Pillow, P.C.; Amato, R.J.; Hutchinson, L.P.; Sweeney, A.M.; Strom, S.S. A case-control study of diet and testicular carcinoma. Nutr. Cancer 1999, 34, 20-26.

124. Markaki, I.; Linos, D.; Linos, A. The influence of dietary patterns on the development of thyroid cancer. Eur. J. Cancer 2003, 39, 1912-1919.

125. Grant, W.B. Update on evidence that support a role of solar ultraviolet-B irradiance in reducing cancer risk. Anti-Cancer Agents Med. Chem. 2013, 13, 140-146.

126. Bellack, N.R.; Koehoorn, M.W.; MacNab, Y.C.; Morshed, M.G. A conceptual model of water's role as a reservoir in Helicobacter pylori transmission: A review of the evidence. Epidemiol. Infect. 2006, 134, 439-449.

127. Ferreccio, C.; Smith, A.H.; Duran, V.; Barlaro, T.; Benitez, H.; Valdes, R.; Aguirre, J.J.; Moore, L.E.; Acevedo, J.; Vasquez, M.I.; et al. Case-control study of arsenic in drinking water and kidney cancer in uniquely exposed Northern Chile. Am. J. Epidemiol. 2013, 178, 813-818.

128. Lopez-Abente, G.; Garcia-Perez, J.; Fernandez-Navarro, P.; Boldo, E.; Ramis, R. Colorectal cancer mortality and industrial pollution in Spain. BMC Public Health 2012, 12, 589.

129. Petti, S.; Masood, M.; Scully, C. The magnitude of tobacco smoking-betel quid chewing-alcohol drinking interaction effect on oral cancer in South-East Asia. A meta-analysis of observational studies. PLoS One 2013, 8, e78999.

\section{Appendix}

The 87 countries with level 1 or 2 quality and life expectancy for males in $1990>60$ years: Albania, Argentina, Armenia, Australia, Austria, Azerbaijan, Bahamas, Barbados, Belarus, Belgium, Belize, Brazil, Brunei, Bulgaria, Canada, Columbia, Costa Rica, Croatia, Cuba, Cyprus, Czech Republic, Denmark, Dominican Republic, Ecuador, Egypt, El Salvador, Estonia, Fiji, Finland, France, Georgia, Germany, Greece, Guatemala, Guyana, Hungary, Iceland, Iran, Ireland, Israel, Italy, Jamaica, Japan, Kazakhstan, Korea (Republic), Kuwait, Kyrgyzstan, Latvia, Lithuania, Luxembourg, 
Macedonia, Malaysia, Mauritius, Mexico, Mongolia, Netherlands, New Zealand, Nicaragua, Norway, Panama, Paraguay, Peru, Philippines, Poland, Portugal, Romania, Russia, Slovakia, Slovenia, South Africa, Spain, Sri Lanka, Suriname, Swaziland, Sweden, Switzerland, Syria, Tajikistan, Trinidad and Tobago, Turkey, Turkmenistan, Ukraine, United Kingdom, United States, Uruguay, Uzbekistan, Venezuela.

The other 70 countries: Algeria, Angola, Bangladesh, Benin, Bolivia, Botswana, Burkina Faso, Burundi, Cambodia, Cameroon, Cape Verde, Central African Republic, Chad, Chile, China, Comoros, Djibouti, Ethiopia, French Polynesia, Gabon, Gambia, Ghana, Guadeloupe, Guinea, Guinea-Bissau, Honduras, India, Indonesia, Iraq, Ivory Coast, Jordan, Kenya, Laos, Lebanon, Lesotho, Libya, Madagascar, Maldives, Mali, Malta, Martinique, Mauritania, Moldova, Morocco, Mozambique, Myanmar, Namibia, Nepal, New Caledonia, Niger, Nigeria, Pakistan, Papua New Guinea, Reunion, Saudi Arabia, Senegal, Sierra Leon, Solomon Islands, Sudan, Tanzania, Thailand, Togo, Tunisia, Uganda, United Arab Republic, Vanuatu, Viet Nam, Yemen, Zambia, Zimbabwe.

(C) 2013 by the authors; licensee MDPI, Basel, Switzerland. This article is an open access article distributed under the terms and conditions of the Creative Commons Attribution license (http://creativecommons.org/licenses/by/3.0/). 